\title{
Towards a Model for Inclusive Healthcare Access post COVID-19
}

\author{
Paras Bhatt \\ The University of Texas \\ at San Antonio \\ paras.bhatt@utsa.edu
}

\author{
Oluwafemi Akanfe \\ The University of Texas at \\ San Antonio \\ oluwafemi.akanfe@utsa.edu
}

\author{
Yuanxiong Guo \\ The University of Texas \\ at San Antonio \\ yuanxiong.guo@utsa.edu
}

\author{
Yanmin Gong \\ The University of Texas \\ at San Antonio \\ yanmin.gong@utsa.edu
}

\begin{abstract}
The devastation caused by the COVID-19 pandemic has exposed years of cyclic inequalities faced by disadvantaged and minority communities. Unequal access to healthcare and a lack of financial resources further exacerbates their suffering, especially during a pandemic. In such critical conditions, information technology-based healthcare services can be an efficient way of increasing access to healthcare for these communities. In this paper, we put forward a decision model for guiding the distribution of IT-based healthcare services for racial minorities. We augment the Health Belief Model by adding financial and technology beliefs. We posit that financial inclusion of minority populations increases their ability to access technology and, by extension, IT-based healthcare services. Financial inclusion and the use of secure private technologies like federated learning can indeed enable greater access to healthcare services for minorities. Therefore, we incorporate financial, health, and technology tools to develop a model for equitable delivery of healthcare services and test its applicability in different use-case scenarios.
\end{abstract}

\section{Introduction}

The COVID-19 pandemic exposed some of the grave inequalities that minorities face when trying to access healthcare facilities [1]. During the pandemic, regions with larger minority populations have suffered more than others because of an acute lack of medical infrastructure [2]. The pandemic has also severely affected the mental health of people [3], which has given rise to a critical need for telehealth and online health counseling services [4]. In spite of this pressing need for telehealth services, people are still wary of new technologies and have several privacy concerns about sharing their personal health information online. To tackle this challenge in a post COVID-19 world, we propose an extension to the health belief model that incorporates financial inclusion, technology usage, and user privacy to build an augmented healthcare delivery model that can improve healthcare access for minorities and economically disadvantaged people.
We posit that when people have greater financial resources and are confident that their personal health information is secure, they are more likely to access healthcare facilities in general.

Health outcomes are shaped by people's beliefs in the efficacy of healthcare facilities as well as their perception of health risks. The Health Belief Model (HBM) [5] states that people's behavior is linked to their beliefs and perceptions about health-related threats such as the susceptibility to catching a virus. Researchers have used HBM to understand, explain, and predict people's health-related behaviors. It has been used to study various chronic diseases, child maladies, and even understand smoking-related behaviors. Therefore, we believe HBM provides a strong theoretical framework to study population-level health behavior, especially during a time of medical crisis like the current pandemic.

Over the years, hospitals and medical institutions have adopted IT at a rapid pace and currently use it to deliver many of its services [6]. From telehealth to online counseling, IT has redefined how people access healthcare facilities. There have also been significant advances in both hardware and software, such as the Internet of Medical Things (IoMT) devices and artificial intelligence algorithms, respectively. IT has significantly contributed to the development of new ways to detect and diagnose medical conditions. Hence, for our proposed model, it is important that we consider people's technology beliefs in conjunction with their health beliefs.

However, with the growth in healthcare technology usage, there has been a significant increase in user privacy concerns as well [7]. For example, wearable sensors can now continuously monitor patient activity [8] and track people through their smartphones [9]. As a result, highly sensitive information can be shared with others using such technology. This gives rise to legitimate user privacy concerns and ultimately affects the widespread adoption of technology resources. This also inhibits people from accessing IT healthcare services. Even though data from health technology devices can be used to train machine learning models that predict the presence of diseases [10], the risks to people who share 
such data are considerable. The shared data is often communicated to central servers, databases, and data repositories. There is a chance that such data may get compromised in the transmission process.

To address this issue, recent developments in deep learning can be leveraged, such as the federated learning (FL) model, which is a resilient privacypreserving model that does not need access to user data. The use of tools such as FL models for enhancing privacy protection of users' health data can ultimately strengthen people's technology beliefs and also enable wider access to healthcare services for minorities. We posit that IT resources like FL increase the technology beliefs of people and motivate them to access a greater level of healthcare services.

In addition to technology beliefs, financial inclusion is another metric linked to greater healthcare access. The link between greater financial inclusion and higher levels of healthcare facilities usage has been well documented in social research [11]. This is because when people have more financial resources at their disposal, they are likely to invest in more and better healthcare services. This is especially true for both minorities and economically disadvantaged populations.

We propose a conceptual model that augments the HBM into the Financial Inclusion, Health and Technology Belief (F-HAT) Model that incorporates additional dimensions of financial inclusion and technology usage. Through a healthcare use case scenario, we showcase that increased access to health facilities is driven by advances in people's financial and technological resources. From the use case results, we also present some propositions that can guide future research into the confluence of technology, finance, and healthcare.

We pursue the following research questions in our study: 1. How can technology be used to increase access to healthcare services for minorities? 2. How does financial inclusion affect healthcare access and technology access? 3. What scenarios can be developed to demonstrate the importance of a shared architecture incorporating health and technology?

The next section includes a survey of the literature of the constructs discussed in this paper. The following section includes an explanation of the conceptual research model based on the health belief model, followed by the section explaining the study methodology and use case scenarios. This is followed by the results section, in which we put forth certain propositions that can guide future research and development of such shared architecture with health and technology resources. Finally, in the conclusion section, the future continuation of this work is presented, and the contributions, implications, and limitations of this study are discussed at length.

\section{Literature Review}

\subsection{Financial Inclusion}

Researchers have found that financial inclusion and human development is interlinked [12]. When more people are connected to financial systems, they can avail greater medical, housing, and educational resources. Financial inclusion increases the ability of disadvantaged and minority population to tap into financial systems. People can obtain health insurance, apply for mortgages and pursue higher education through increased financial inclusion. Based on the relationship between financial development and economic growth, Sarma [13] proposed an index of financial inclusion that serves as an empirical indicator of the level of financial inclusion seen in the community. Demirguc-Kunt and Klapper [14] have put forward and analyzed the Global Financial Inclusion (Global Findex) Database that indicates how people across the world save, borrow, make payments, and manage risk. These indicators are important criteria for analyzing the level of access to financial resources that people in an area can afford. Through such indicators, we can not only define how financial inclusion affects healthcare access but can also model their usage levels as a function of their financial resources.

Studies have shown that financial inclusion programs are affected by demographic differences [15]. The effect of financial inclusion for minorities can be significant since the large sector of the population has a lower median household income than their majority counterparts. Al-Hanawi et al. [16] noted that financial exclusion leads to several obstructions for people who intend to access healthcare facilities even in countries with a high per capita income. Countries with high human development can still have certain areas and populations that have been historically disadvantaged and excluded from gaining equitable access to healthcare and financial resources. Financial inclusion not only leads to greater access to healthcare but also to increased methods of managing finances such as mobile money, which has been shown to be particularly useful in rural areas [17]. This is important to consider for nations with a large percentage of their population residing in remote and rural areas with limited access to healthcare facilities. Lumsden [18] has also noted that an increase in financial inclusion levels can spur increased healthcare and technology resources usage. With the advancements in the field of 
AI and the widespread adoption of wearable and remote health technology, financial inclusion will indeed play a vital role in ensuring equitable access to healthcare facilities for minorities and economically disadvantaged people in the future.

\subsection{Technology Belief}

Technology has been widely used in the healthcare sector for a variety of purposes, such as diagnosing diseases [19] to predict vulnerable populations that are at risk of contracting certain illnesses [20]. FL as an advanced ML technique has gained popularity in recent years. Fl is markedly different from ML, and this difference is manifested in how data is handled in each of these techniques [21]. For ML, data needs to be stored in a central database and the learning algorithm is trained on the stored data. For FL, there is no need for a central repository to store data. The learning algorithm runs locally on individual devices such as wearable medical devices that generate real-time data. These devices have network connections, power, and hardware specifications [22], but for FL each of them can run training models at differing speeds and latency. The type of data that is collected from such devices can be related to activity monitoring (counting the number of steps), user location (GPS coordinates), or sensors information (heart rate of the user). We can leverage the distributed nature of FL to ensure people's privacy is protected and their sensitive health data is not exchanged or transmitted. Such privacy protections can strengthen the technology beliefs of people who can resultantly utilize healthcare technology with much more confidence. FL has been growing in importance in recent years and is the future of digital health [23]. It can be used for training models on a high volume of data that are collected by modern healthcare organizations. FL can not only ensure data interoperability but also enhance the privacy of data. Personalized FL models are built on individual differences between users and prevent sensitive information from being exposed [24]. Such models can be generalized and applied to a large section of the population. FL also can be combined with crossplatform technologies such as Blockchain to ensure strong privacy protections when detecting the spread of COVID-19 [25]. Studies have shown how FL can be used to train a model without breaking data privacy laws and guidelines such as HIPAA [26]. Thus, FL ensures that users' privacy is not violated, and their personally information is not compromised. Such privacy protections can significantly increase people's confidence and technology beliefs regarding healthcare facilities utilization.

\subsection{Health Belief Model (HBM)}

The health belief model can be effectively summarized in the following example. A person may quit smoking only if they believe that quitting would positively affect their health and that they are capable of actually quitting smoking. In the example, quitting smoking is the behavior, the person's expectation of having better health is the outcome, and the capability of quitting is the efficacy [5]. All three are important for people to believe that indulging in any healthcare activity will help them live a healthier life. The key tenet of the HBM is that people's health behavior can be influenced by certain cues to action and depends on their demographic and psychological characteristics, known as the modifying factors, and their individual beliefs regarding health [27]. The perceived susceptibility refers to the likelihood of getting a disease, whereas the perceived severity refers to the seriousness of the disease. The perceived benefits indicate the positive impacts that may come from performing a healthy behavior such as quitting smoking. On the other hand, the perceived barriers relate to the negative outcomes that may come from the behavior, such as withdrawal symptoms if the person is a chain smoker. The cues to action include external influences that can motivate the person to perform the behavior, such as anti-smoking warnings on cigarette packs. Finally, perceived self-efficacy refers to the person's belief in their willpower and ability to actually quit smoking. The health belief model from Champion and Skinner [27] is shown below in Figure 1, along with the metrics in the model shown in Table 1.

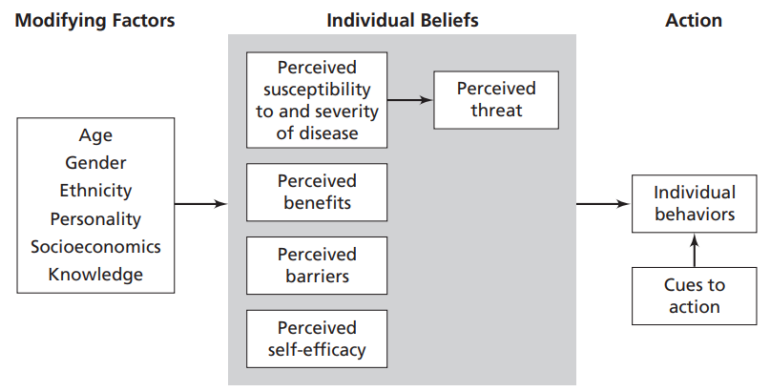

Figure 1. Health Belief Model

\begin{tabular}{|l|l|}
\hline Term & Meaning \\
\hline $\begin{array}{l}\text { Perceived } \\
\text { Susceptibility }\end{array}$ & $\begin{array}{l}\text { Likelihood of contracting a disease } \\
\text { or illness. }\end{array}$ \\
\hline $\begin{array}{l}\text { Perceived } \\
\text { Severity }\end{array}$ & $\begin{array}{l}\text { Seriousness of the disease or the } \\
\text { non-performance of behavior. }\end{array}$ \\
\hline $\begin{array}{l}\text { Perceived } \\
\text { Benefits }\end{array}$ & $\begin{array}{l}\text { Benefits from the performance of a } \\
\text { health behavior. }\end{array}$ \\
\hline $\begin{array}{l}\text { Perceived } \\
\text { Barriers }\end{array}$ & $\begin{array}{l}\text { Inhibitors to performance of health } \\
\text { behavior. }\end{array}$ \\
\hline
\end{tabular}




\begin{tabular}{|l|l|}
\hline $\begin{array}{l}\text { Perceived } \\
\text { Self-efficacy }\end{array}$ & $\begin{array}{l}\text { Confidence in the ability to } \\
\text { successfully perform a behavior. }\end{array}$ \\
Cues to action & $\begin{array}{l}\text { External motivating factors } \\
\text { encouraging behavior performance. }\end{array}$ \\
\hline
\end{tabular}

Table 1. Health Belief Model Metrics

Researchers have shown that though HBM can be used to influence people to perform behaviors that are healthy for them, these may not influence everyone in the same way. Sayegh and Knight [28] have shown how stigma associated with certain diseases and medical conditions can inhibit people from performing certain behaviors, such as getting a doctor's opinion about their conditions. In a similar vein, the potential of their private health information being leaked or compromised is another example of how individuals may not always perform certain beneficial health behavior. People may reject the use of technology if their belief in its efficacy is not strong enough. In contrast, if people believe that a technology preserves their privacy, it can encourage them to participate in the health behavior associated with the use of such technology. The HBM is a versatile model and has been used in a wide variety of contexts to study behaviors related to pandemics [29], vaccinations [30], and even psychometric testing [31]. Therefore, HBM is a suitable framework for this study and can be used to model how technology and financial inclusion metrics can contribute to increased access to healthcare facilities and services for minorities and economically disadvantaged populations.

To derive our new healthcare delivery model, we use HBM as a theoretical base for analyzing how individuals perceive their chances of being affected by an adverse health outcome. The HBM provides a framework for analyzing the health-related motivations and beliefs of people. It helps us to understand how people can be reasoned with and persuaded to access more healthcare resources. In addition to health beliefs, technology beliefs are equally important as technology usage can motivate people to explore new avenues for accessing healthcare services. Checking in on a doctor's appointment through telehealth or sharing their vital signs and symptoms through wearable devices can enable people to regularly check their health status. Further, health and technology beliefs can be strengthened by using technology with strong privacy protections. In this regard, using techniques such as FL can enhance the perceived benefits of technology for people and enable cues to action that ultimately lead them to access more healthcare services in general. Thus, using these beliefs, we explain our proposed model in the next section.

\section{Financial Inclusion, Health and Technology Belief (F-HAT) Model}

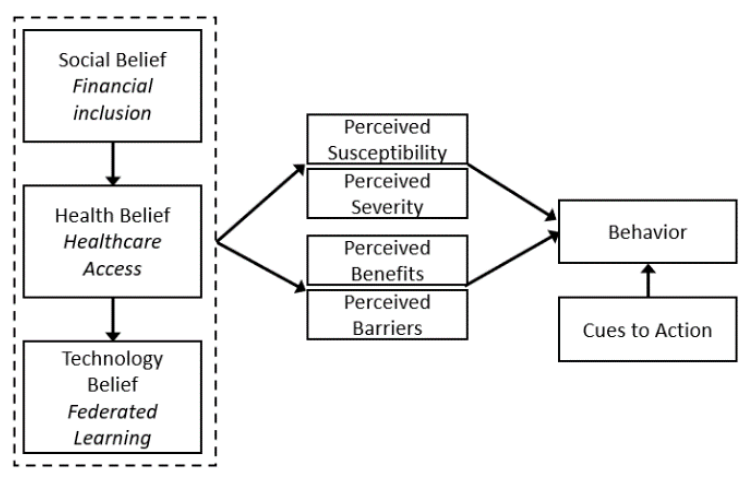

Figure 2. F-HAT Model

Our proposed F-HAT model shown in Figure 2 takes into account the double interaction effect of greater financial inclusion on the higher level of healthcare access and consequently on the increased usage of technology. Also, the three beliefs have a chronological multiplicative effect with social beliefs giving rise to stronger health beliefs and greater technology beliefs. The three distinct beliefs of social, health, and technology have certain costs and benefits associated with them. In terms of costs, these increased levels of beliefs can be associated with lower perceived susceptibility and perceived severity. In contrast, the increased levels of beliefs can be associated with greater perceived benefits lower perceived barriers. Based on these costs and benefits, the individual engages in a particular health behavior. This behavior can also be influenced by certain factors that are outside the purview of the three beliefs. These cues are a callout to the individual to perform a certain behavior but are not directly responsible for the performance. These cues may arise because of the different beliefs and are specific to the type of belief in question. For instance, cues to action relating to social beliefs are related to how the minority population can be included in the financial ecosystem, and cues relating to technology beliefs are related to how various technology features can serve as the motivation for people to access remote healthcare facilities and services.

From our literature review, we establish that FL can ensure data privacy by training models on data collected through remote health monitoring devices such as sensors or smartphones. We have also noted how financial inclusion enables greater access to healthcare resources which ensures greater technology utilization. Furthermore, greater technology use can foster the adoption of the latest technologies such as 
FL. Therefore, financial inclusion serves as a catalyst for enabling greater healthcare and technology access.

\section{Methodology}

\subsection{Federated Learning Model}

In FL, the devices train models locally on the data stored on them and communicate the trained local models with the central server, which aggregates all the different local models into a global model. The absence of data transmission to the central server essentially results in preserving user data privacy. Figure 3 depicts the architecture for a FL scenario involving different smart devices holding various sensitive private information about their users. This information may be in the form of location data such as GPS coordinates or medical data such as heart rate.

For instance, in a medical study conducted to detect arrhythmia, there are a number of participants who willingly provide their personal data to train a FL learning model that can predict the occurrence of irregular heartbeats and can send active interventions to caregivers of users who might be experiencing such irregular heartbeats. To protect the privacy of each user's data, their devices train a learning model locally on their own data. Once training is completed, only the trained model is transmitted to the central server rather than the entire personal data of the users. The models trained on individual devices are called local models. The many local models are then aggregated to form a central learning model known as the global model. This aggregation of multiple local models is the essence of FL scenarios.

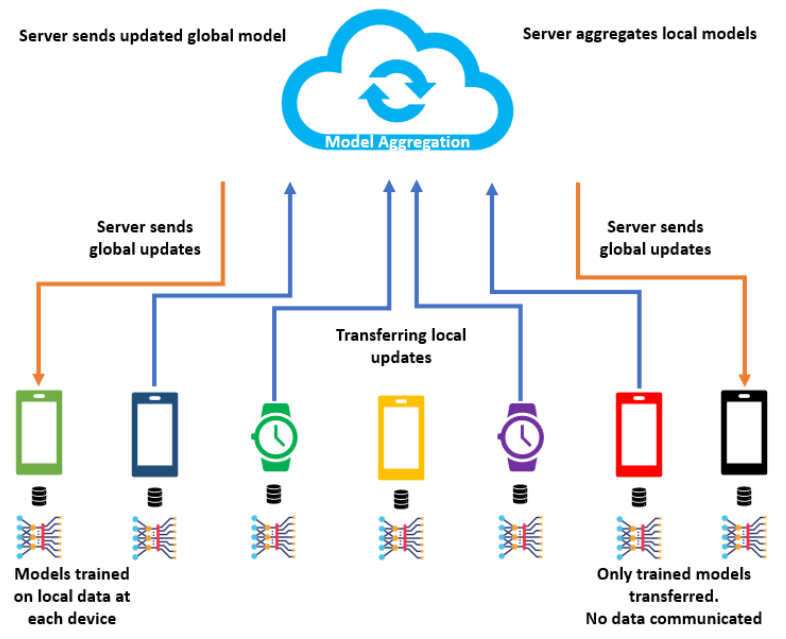

Figure 3. FL Architecture

The multiple devices that train the different models on locally available data ensure the privacy of user data. This behavior of the FL model can increase the technology beliefs of users as they become confident that their data is secure. The technology beliefs are enabled through the health beliefs that if personalized data is used to build a prediction model it can benefit the general population facing a particular medical condition. Since users' heart rate is a sensitive piece of information, as it can be used to decipher the health status of an individual, it should be protected through technology resources such as FL techniques.

\subsection{Use Case Scenarios}

We explain two use case scenarios to validate the F-HAT model. These use cases are based on Rieke et al.'s [33] work who explain how FL affects patients' health outcomes. They state that FL lowers the hurdles for becoming a data donor. This is because patients are reassured that their data remains with their own institution and access to their data can be restricted instantly at their request. They also note that when a technology like FL is implemented on a large scale, it positively impacts the technology usage by both hospitals and patients. Thus, it increases both health beliefs and technology beliefs. In the following use cases, we modulate how social beliefs interact with health and technology beliefs and determine if this interaction results in the performance of certain health behaviors.

\subsubsection{Higher-order Beliefs and Behavior}

In our first use case a person suffers from depression but does not have health insurance coverage and is afraid to seek online counseling because of privacy concerns.

\begin{tabular}{|l|l|l|l|l|}
\hline & $\begin{array}{l}\text { Social } \\
\text { Belief }\end{array}$ & $\begin{array}{l}\text { Health } \\
\text { Belief }\end{array}$ & $\begin{array}{l}\text { Tech } \\
\text { Belief }\end{array}$ & Behavior \\
\hline $\begin{array}{l}\text { Social } \\
\text { Belief }\end{array}$ & $\checkmark$ & $x$ & $\begin{array}{l}\text { Not } \\
\text { Performed }\end{array}$ \\
\hline $\begin{array}{l}\text { Health } \\
\text { Belief }\end{array}$ & $\checkmark$ & $\checkmark$ & Performed \\
\hline $\begin{array}{l}\text { Tech } \\
\text { Belief }\end{array}$ & $\checkmark$ & $x$ & $\begin{array}{l}\text { Not } \\
\text { Performed }\end{array}$ \\
\hline $\begin{array}{l}\text { Behavi- } \\
\text { or }\end{array}$ & $\begin{array}{l}\text { Perfor- } \\
\text { med }\end{array}$ & $\begin{array}{l}\text { Not } \\
\text { Perfor- } \\
\text { med }\end{array}$ & $\begin{array}{l}\text { Not } \\
\text { Perfor- } \\
\text { med }\end{array}$ \\
\hline \multicolumn{4}{|c|}{ Belief Present } & \multicolumn{3}{|c|}{ Belief Absent } \\
\hline
\end{tabular}

Table 2. F-HAT Model Belief Scenarios 
This scenario shown in Table 2 above, deals with different beliefs in the F-HAT model which must exist if a certain health behavior has to be performed. For example, for the depressed person to seek online counseling (health belief), she/he must possess means to access health insurance (social belief) and be willing to share their information on telehealth platforms (technology belief). From Table 2, we observe that out of six outcomes, two result in the behavior being performed. These two instances are when all three beliefs are concurrently held by a person. In such a situation, the depressed person will actually seek online counseling only if: she/he is properly insured; she/he believes that telehealth counseling has the same privacy protections as regular counseling; and she/he thinks that online counseling will help her/him to overcome depression.

\begin{tabular}{|c|c|c|c|c|c|}
\hline Low High & Threats & Benefits & Barriers & Self-efficacy & Behavior \\
\hline Threats & & $\mathrm{O}$ & $\mathrm{O}$ & $\mathrm{O}$ & $\begin{array}{l}\text { Performed } \\
\left(\mathbf{L}_{\mathbf{t}} \mathbf{H}_{\mathbf{b}} \mathbf{H}_{\mathbf{r}} \mathbf{H}_{\mathbf{s}}\right)\end{array}$ \\
\hline Benefits & $\mathrm{O}$ & & $\mathrm{O}$ & $\mathrm{O}$ & $\begin{array}{l}\text { Not Performed } \\
\left(\mathbf{L}_{\mathbf{b}} \mathbf{H}_{\mathbf{t}} \mathbf{H}_{\mathbf{r}} \mathbf{H}_{\mathbf{s}}\right)\end{array}$ \\
\hline Barriers & $\mathrm{O}$ & $\mathrm{O}$ & & $\mathrm{O}$ & $\begin{array}{l}\text { Performed } \\
\left(\mathbf{L}_{\mathbf{r}} \mathbf{H}_{t} \mathbf{H}_{b} \mathbf{H}_{\mathbf{s}}\right)\end{array}$ \\
\hline Self-efficacy & $\mathrm{O}$ & $\mathrm{O}$ & $\mathrm{O}$ & & $\begin{array}{l}\text { Not Performed } \\
\left(\mathbf{L}_{\mathbf{s}} \mathbf{H}_{\mathbf{t}} \mathbf{H}_{\mathbf{b}} \mathbf{H}_{\mathbf{r}}\right)\end{array}$ \\
\hline Behavior & $\begin{array}{l}\text { Not Performed } \\
\left(\mathbf{H}_{\mathbf{t}} \mathbf{L}_{\mathbf{b}} \mathbf{L}_{\mathbf{r}} \mathbf{L}_{\mathbf{s}}\right)\end{array}$ & $\begin{array}{l}\text { Performed } \\
\left(\mathbf{H}_{\mathbf{b}} \mathbf{L}_{\mathbf{t}} \mathbf{L}_{\mathbf{r}} \mathbf{L}_{\mathbf{s}}\right)\end{array}$ & $\begin{array}{l}\text { Not Performed } \\
\left.\mathbf{H}_{\mathbf{r}} \mathbf{L}_{\mathbf{t}} \mathbf{L}_{\mathbf{b}} \mathbf{L}_{\mathbf{s}}\right)\end{array}$ & $\begin{array}{l}\text { Performed } \\
\left(\mathbf{H}_{\mathbf{s}} \mathbf{L}_{\mathbf{t}} \mathbf{L}_{\mathbf{b}} \mathbf{L}_{\mathbf{r}}\right)\end{array}$ & \\
\hline
\end{tabular}

Table 3. F-HAT Model Individual Beliefs

\subsubsection{Lower-order (Individual) Beliefs and Behavior}

In the second scenario shown in Table 3 above, we continue with the person with depression but now our criteria include individual factors that affect decision to perform a health-related behavior. This involves analyzing high and low levels of factors that influence a particular situation, such as the one described in our scenario. In Table 3, we model the high and low levels of individual factors that affect the decision to perform a health-related behavior. There are two outcomes in this use case. First, positive outcomes are: high level of benefits; high level of selfefficacy; low level of threats; and low level of barriers. Second, negative outcomes are: high level of threats; high level of barriers; low level of benefits; and low level of self-efficacy. The decision criteria is as follows: if the number of positive outcomes exceed the negative outcomes, then the health behavior is performed.

In the first row, we have a low level of threats combined with a high level of benefits, high barriers, and high self-efficacy. Since the positive outcomes are greater, we perform the health-related behavior. In the second row, we have a low level of benefits combined with a high level of threats, a high level of barriers, and a high level of self-efficacy. Since the negative outcomes outweigh the positive, the health behavior is not performed. In the third row, we have a low level of barriers followed by a high level of threats, a high level of benefits, and a high level of self-efficacy. Similarly, the positive outcomes outweigh the negative, and hence the health behavior is performed. In the last row, the self-efficacy is low as compared to high levels of threats, benefits, and barriers, thus resulting in the health behavior not being performed.

Next, we move to the columns, first where we have a high level of threats combined with a low level of benefits, barriers, and self-efficacy. Since the positive outcomes are less, we make a similar decision in line with our decision criteria, and thus the health behavior will not be performed in this scenario. Second, we have a high level of benefits along with low levels of threats, barriers, and self-efficacy. Here the positives are greater than the negative, and hence the health behavior is performed. Third, we have high levels of barriers with a low level of threats, benefits, and self-efficacy. The health behavior will not be performed in this case in consistence with our decision criteria. Finally, the high level of self-efficacy is combined with a low level of threats, benefits, and barriers. Since in this scenario, the positives outweigh the negatives, the health behavior will be performed. 


\section{Discussion}

Based on our use case scenarios and the greater level of privacy protections afforded by FL, we present a set of propositions that can guide further validation of the F-HAT model and encourage future research in the domain of equitable healthcare access models. From the analysis of higher-order beliefs related to financial inclusion, healthcare access, and the use of federated learning, we can distinguish various scenarios in which a health-related behavior will be easy for a person to perform. This analysis is important since minorities and economically disadvantaged populations are not able to access financial resources, which sets off a chain reaction that limits their ability to access healthcare facilities, interact with technological advancements in healthcare services, and in turn develop stronger technology beliefs for the said technology. The different scenarios related to higher-order beliefs in Table 2 can be condensed into two main decision criteria.

First is the case where all three beliefs are present in a scenario. People that have a strong social belief in their ability to access financial resources, through which they can access more healthcare services, and if they believe that their privacy is protected while using technology, then they perform health-related behaviors that are beneficial to them. For example, the smoker is more likely to quit smoking if she/he can afford addiction support programs (financial inclusion), have access to online health counseling for dealing with withdrawal symptoms (healthcare access), and believe that the privacy of her/his data related to addiction behavior is preserved and will not be shared with anyone else such as her/his insurance company or employer (federated learning). Based on these findings, we put forth the following proposition:

P1 - When people have higher levels of three beliefs - social, health and technology - they are more likely to perform a health-related behavior that can benefit them.

Second is the case where two of the beliefs might be high but the third one is not. In these situations, people may possess stronger beliefs for two of the three combinations of beliefs. A person may possess stronger social belief and health belief but may not believe that the technology adequately protects their privacy which would lead to the non-performance of an otherwise beneficial health-related behavior. In another case, a person that has strong social belief and an affinity to use technology to monitor their health, but does not possess strong healthcare beliefs, such as access to advanced healthcare required for monitoring chronic diseases, will also result in non-performance of a beneficial health-related behavior. A key thing to note is that in both cases, social beliefs are necessary as a precondition for other beliefs to exist since they act as a catalyst for other types of beliefs for people. For example, suppose the smoker has access to financial resources for rehabilitation (financial inclusion) and believes that her/his healthcare information is secure (technology belief), but does not have access to online health counseling (healthcare access), which is important to develop the willpower needed to quit smoking. In that case, the smoker will not engage in the performance of the beneficial health behavior of quitting smoking.

Therefore, in the above scenario social beliefs relate to the ability of a person to have access to necessary financial resources needed to overcome a negative health behavior such as addiction. These financial resources can be in terms of insurance coverage that covers expenses for addiction treatments like rehabilitation. The presence of social beliefs increases people's health beliefs because when they get the necessary support to treat their addiction then, they are more confident in their ability to give it. Thus, the presence of higher social beliefs fosters greater health beliefs which ultimately result in the performance of a beneficial health related behavior like giving up smoking. Based on these findings, we put forth the following proposition:

P2 - Consistent levels of all three beliefs - social, health, technology - are needed for people to perform a health-related behavior that can benefit them.

Table 4 captures the different outcomes that can take exist when health-related behavior performance is based on the higher order beliefs of people.

\begin{tabular}{|l|l|}
\hline Outcomes & Behavior \\
\hline $\begin{array}{l}\text { Social belief + Health belief }- \text { Tech } \\
\text { belief }\end{array}$ & $\begin{array}{l}\text { Not } \\
\text { performed }\end{array}$ \\
\hline $\begin{array}{l}\text { Social belief + Health belief + Tech } \\
\text { belief }\end{array}$ & Performed \\
\hline $\begin{array}{l}\text { Health belief + Social belief + Tech } \\
\text { belief }\end{array}$ & Performed \\
\hline $\begin{array}{l}\text { Health belief + Social belief - Tech } \\
\text { belief }\end{array}$ & $\begin{array}{l}\text { Not } \\
\text { Performed }\end{array}$ \\
\hline $\begin{array}{l}\text { Tech belief + Social belief }- \text { Health } \\
\text { belief }\end{array}$ & $\begin{array}{l}\text { Not } \\
\text { Performed }\end{array}$ \\
\hline $\begin{array}{l}\text { Tech belief - Social belief + Health } \\
\text { belief }\end{array}$ & $\begin{array}{l}\text { Not } \\
\text { Performed }\end{array}$ \\
\hline
\end{tabular}

Table 4. Higher-order Beliefs Outcomes

Based on Table 4, a decision model can be implemented to determine when a person is likely to engage in a health-related behavior that benefits them. For example, in the case of COVID-19 vaccine 
hesitancy, our model can be used to determine how to encourage people to get vaccinated. If people are fearful of the side effects of the vaccine, then policymakers should focus on strengthening people's health beliefs. They can achieve this by promoting the efficacy of the vaccine and citing its effectiveness level (e.g., Moderna and Pfizer vaccines are almost $95 \%$ effective ${ }^{1}$ ). If people are fearful of the technology used in the vaccine (mRNA - messenger RNA), then policymakers should focus on strengthening people's technology beliefs. They can achieve this by increasing awareness about the reasons why a vaccine was developed in such a short time (e.g., mRNA instructs cells to produce copies of virus whereas traditional vaccines induce an immune response ${ }^{2}$ ). Finally, if people do not get vaccinated because their insurance does not cover vaccination expenses or if there are no vaccination centers near them, then policymakers should focus on strengthening the social beliefs. They can achieve this by increasing federal insurance coverage or ask insurance providers to enhance coverage or even open mobile vaccination clinics that visit medically underserved areas (MUAs).

Moving to lower order beliefs, from the analysis of the individual beliefs related to people's perception of situations, we can distinguish various scenarios in which performing a health-related behavior will become easy for a person. These metrics are important to analyze as individual and demographic differences are an integral part of the HBM and can help to understand how people access social, healthcare, and technological resources. The different scenarios related to lower-order individual beliefs in Table 3 can be condensed into an additive decision criterion based on the combination of high and low levels of perceived individual beliefs. In this regard, there are four positive outcomes associated with the performance of health-related behavior. First, when people perceive high benefits from the performance of the behavior. Second, when they perceive they have a high level of self-efficacy in performing the behavior and are confident that they can successfully perform it. Third, when they perceive low levels of threats associated with the performance of a particular behavior. Fourth, when they perceive low levels of barriers exist that might hinder the performance of the health-related behavior. Also, there exist four negative outcomes that are the polar opposite of the positive outcomes. Specifically, they are low benefits associated with health-related behavior performance and low levels of self-efficacy among people regarding such performance. Along with these, a high level of barriers

\footnotetext{
${ }^{1}$ https://www.yalemedicine.org/news/covid-19-vaccinecomparison
}

that hinder the performance of a behavior and the higher level of threats associated with a health-related behavior are also negative outcomes. Table 5 presents the combination of these additive outcomes that determine whether a person performs a health-related behavior or not. A person performs the behavior if the positive outcomes outweigh the negative outcomes.

\begin{tabular}{|l|l|}
\hline Outcomes & Behavior \\
\hline $\begin{array}{l}\text { High threats + low benefits + low } \\
\text { barriers }+ \text { low self-efficacy }\end{array}$ & $\begin{array}{l}\text { Not } \\
\text { Performed }\end{array}$ \\
\hline $\begin{array}{l}\text { Low threats + high benefits +high } \\
\text { barriers }+ \text { high self-efficacy }\end{array}$ & Performed \\
\hline $\begin{array}{l}\text { High benefits + low threats + low } \\
\text { barriers }+ \text { low self-efficacy }\end{array}$ & Performed \\
\hline $\begin{array}{l}\text { Low Benefits }+ \text { high threats + high } \\
\text { barriers }+ \text { high self-efficacy }\end{array}$ & $\begin{array}{l}\text { Not } \\
\text { Performed }\end{array}$ \\
\hline $\begin{array}{l}\text { High barriers }+ \text { low threats }+ \text { low } \\
\text { benefits }+ \text { low self-efficacy }\end{array}$ & $\begin{array}{l}\text { Not } \\
\text { Performed }\end{array}$ \\
\hline $\begin{array}{l}\text { Low barriers }+ \text { high threats }+ \text { high } \\
\text { benefits }+ \text { high self-efficacy }\end{array}$ & Performed \\
\hline $\begin{array}{l}\text { High self-efficacy }+ \text { low threats }+ \text { low } \\
\text { benefits }+ \text { low barriers }\end{array}$ & Performed \\
\hline $\begin{array}{l}\text { Low self-efficacy }+ \text { high threats }+ \text { high } \\
\text { benefits }+ \text { high barriers }\end{array}$ & $\begin{array}{l}\text { Not } \\
\text { Performed }\end{array}$ \\
\hline
\end{tabular}

Table 5. Individual Beliefs Outcomes

Continuing with the example for the smoker, we can explain the possible outcomes related to individual beliefs and perceptions of people. For instance, the smoker would perform a health-related behavior (quitting smoking) that is beneficial for them if she/he perceives that doing so would not result in withdrawal symptoms (low threats) and would significantly help in controlling her/his high blood pressure (high benefits). The smoker might be apprehensive of her/his ability to actually quit smoking if she/he is a chain smoker (high barriers), but her/his confidence in her/his ability and willpower to quit (high selfefficacy) will help the person to give up smoking. Based on these findings, we put forth the following proposition:

P3 - Higher levels of people's positive perception related to their individual beliefs - perceived threats, perceived benefits, perceived barriers, perceived selfefficacy - is associated with a greater likelihood of performing a health-related behavior that can benefit them.
${ }^{2} \underline{\mathrm{https}: / / \mathrm{www} . v u m c . o r g / v i i i i / i n f o g r a p h i c s / h o w-d o e s-m r n a-}$
vaccine-compare-traditional-vaccine 


\section{Conclusion}

We investigate the role of financial inclusion and technology usage in expanding access to healthcare for people, especially the minorities and economically disadvantaged population. Using HBM framework, we build a conceptual model that incorporates the role of financial inclusion and technology usage to define situations in which people are more likely to perform health-related behaviors that benefit their general health. Increasing financial resources and IT usage are both equally important for ensuring equitable delivery of healthcare services [16, 23]. We find similar evidence from our use case scenarios highlighting the critical nature of these two metrics in ensuring access to quality healthcare facilities for all.

In terms of the contribution from this paper, we extend the HBM to incorporate financial inclusion and technology usage as key determinants of whether people will engage in health-related behavior that is beneficial for them. In many situations, we can observe that people knowingly do not engage in such behavior. Smoking and other vices are an example of this behavior but are related to the personal volition of individuals. They choose to indulge in such risky behavior that may affect their health. However, for minorities and economically disadvantaged people, such non-performance of health-related behavior may not be a choice but a necessity. This is true for people that lack the financial resources to access healthcare or the technology resources that can ensure the privacy of their healthcare transactions. Using the F-HAT model proposed in this study, the decision regarding healthcare access and equitable delivery of healthcare services can be made effectively. We also contribute to the cross-domain literature on IT healthcare. Digital health is the future of healthcare services [32], and it is important to understand the motivations of people accessing the online healthcare infrastructure. The FHAT model can serve as a modeling tool to incorporate metrics such as healthcare affordability and intention to use digital technology.

The research implications from this work are manifold: with an extension to the traditional HBM, continued research in further solidifying this work is needed. The allied concepts related to finance and technology can serve as additional branches of the FHAT model and be validated in a wide variety of scenarios ranging from minority and rural healthcare provision to research relating to mental health and addiction. The F-HAT model can also be used as a metric for judging competitive grants that are targeted towards improving people's health outcomes. The practical implications from this study relate to the importance of the F-HAT model for policymakers that can use the insights drawn from the model to build and design social justice programs that target the most vulnerable and disadvantaged sections of society. The interrelated nature of the three beliefs, including social, health, and technology, warrant that welfare programs designed to alleviate the health concerns of people are actually working to benefit the intended population. Our model can also be used as a resource allocation model for ensuring equitable distribution of healthcare and technology services. It can be of particular importance in devising rural health programs in Medically Underserved Areas (MUAs) and Health Professional Shortage Area (HPSAs) ${ }^{3}$

As limitations, we acknowledge the need for using real-world data to empirically test this model, which is addressed in our current ongoing work. Also, we use only a single variable of financial inclusion for denoting social beliefs. Some of the other variables include education and socioeconomic status. However, we note that an increase in financial resources does lead to increased access to education [12], and therefore the effect would be retroactively captured by the financial inclusion metric. Also, our current model only allows for one factor to be set as low/high. We aim to further develop this model in our future work that will incorporates variations in multiple factors. Further, another limitation is that we use FL as the only variable for technology beliefs. We aim to address this in our future work and build a model with a more general view of technology beliefs.

In ongoing work, we use real-world datasets to validate our model and focus on developing FL models that provide healthcare insights from distributed data provided by people which showcases their increasing trust in healthcare technology.

\section{Acknowledgement}

The work of P. Bhatt, Y. Guo, and Y. Gong was partially supported by National Science Foundation under grant number CNS-2029685.

\section{References}

[1] Bhatt, P., Guo, Y., \& Gong, Y. (2021, January). Discounting Lives: Analyzing the causes of Hispanic Adversity raging in the Healthcare sector during Covid19 using Cumulative Inequality Theory. In Proceedings of the 54th Hawaii International Conference on System Sciences (p. 3982). 
[2] Nanda, S. (2020). Inequalities and COVID-19 1. In COVID-19 (pp. 109-123). Routledge.

[3] Pfefferbaum, B., \& North, C. S. (2020). "Mental health and the Covid-19 pandemic," New England Journal of Medicine, 383(6), 510-512.

[4] Li, W., Yang, Y., Liu, Z. H., Zhao, Y. J., Zhang, Q., Zhang, L., ... \& Xiang, Y. T. (2020). "Progression of mental health services during the COVID-19 outbreak in China," International journal of biological sciences, 16(10), 1732.

[5] Rosenstock, I. M., Strecher, V. J., \& Becker, M. H. (1988). Social learning theory and the health belief model. Health education quarterly, 15(2), 175-183.

[6] Lenz, R., \& Reichert, M. (2007). IT support for healthcare processes-premises, challenges, perspectives. Data \& Knowledge Engineering, 61(1), 39-58.

[7] Taylor, C. B., Fitzsimmons-Craft, E. E., \& Graham, A. K. (2020). "Digital technology can revolutionize mental health services delivery: The COVID-19 crisis as a catalyst for change," International Journal of Eating Disorders, 53(7), 1155-1157.

[8] Swayamsiddha, S., \& Mohanty, C. (2020). “Application of cognitive Internet of Medical Things for COVID-19 pandemic," Diabetes \& Metabolic Syndrome: Clinical Research \& Reviews.

[9] Cao, J., Truong, A. L., Banu, S., Shah, A. A., Sabharwal, A., \& Moukaddam, N. (2020). "Tracking and predicting depressive symptoms of adolescents using smartphone-based self-reports, parental evaluations, and passive phone sensor data: development and usability study," JMIR mental health, 7(1), e14045.

[10] Fukazawa, Y., Yamamoto, N., Hamatani, T., Ochiai, K., Uchiyama, A., \& Ohta, K. (2020). "Smartphone-based mental state estimation: A survey from a machine learning perspective," Journal of Information Processing, 28, 16-30.

[11] Hendriks, S. (2019). The role of financial inclusion in driving women's economic empowerment. Development in Practice, 29(8), 1029-1038.

[12] Sarma, M., \& Pais, J. (2011). Financial inclusion and development. Journal of international development, 23(5), 613-628.

[13] Sarma, M. (2008). Index of financial inclusion (No. 215). Working paper.

[14] Demirguc-Kunt, A., \& Klapper, L. (2012). Measuring financial inclusion: The global findex database. The World Bank.

[15] Swamy, V. (2014). Financial inclusion, gender dimension, and economic impact on poor households. World development, 56, 1-15.

[16] Al-Hanawi, M. K., Chirwa, G. C., Kamninga, T. M., \& Manja, L. P. (2020). Effects of Financial Inclusion on Access to Emergency Funds for Healthcare in the Kingdom of Saudi Arabia. Journal of Multidisciplinary Healthcare, 13, 1157.

[17] Bukari, C., \& Koomson, I. (2020). Adoption of Mobile Money for Healthcare Utilization and Spending in Rural Ghana. In Moving from the Millennium to the Sustainable Development Goals (pp. 37-60). Palgrave Macmillan, Singapore.
[18] Lumsden, E. (2018). The Future Is Mobil: Financial Inclusion and Technological Innovation in the Emerging World. Stan. JL Bus. \& Fin., 23, 1.

[19] Sajda, P. (2006). "Machine learning for detection and diagnosis of disease," Annu. Rev. Biomed. Eng., 8, 537-565.

[20] Parthiban, G., \& Srivatsa, S. K. (2012). “Applying machine learning methods in diagnosing heart disease for diabetic patients," International Journal of Applied Information Systems (IJAIS), 3(7), 25-30.

[21] Konečný, J., McMahan, B., \& Ramage, D. (2015). "Federated optimization: Distributed optimization beyond the datacenter," arXiv preprint arXiv:1511.03575.

[22] Li, T., Sahu, A. K., Talwalkar, A., \& Smith, V. (2020). "Federated learning: Challenges, methods, and future directions," IEEE Signal Processing Magazine, 37(3), 50-60.

[23] Rieke, N., Hancox, J., Li, W., Milletari, F., Roth, H. R., Albarqouni, S., ... \& Cardoso, M. J. (2020). "The future of digital health with federated learning," NPJ digital medicine, 3(1), 1-7.

[24] Hu, R., Guo, Y., Li, H., Pei, Q., \& Gong, Y. (2020). Personalized federated learning with differential privacy. IEEE Internet of Things Journal, 7(10), 95309539.

[25] Kumar, R., Khan, A. A., Kumar, J., Zakria, A., Golilarz, N. A., Zhang, S., ... \& Wang, W. (2021). Blockchainfederated-learning and deep learning models for covid19 detection using ct imaging. IEEE Sensors Journal.

[26] Xu, J., Glicksberg, B. S., Su, C., Walker, P., Bian, J., \& Wang, F. (2021). "Federated learning for healthcare informatics," Journal of Healthcare Informatics Research, 5(1), 1-19.

[27] Champion, V. L., \& Skinner, C. S. (2008). The health belief model. Health behavior and health education: Theory, research, and practice, 4, 45-65.

[28] Sayegh, P., \& Knight, B. G. (2013). Cross-cultural differences in dementia: the Sociocultural Health Belief Model. International psychogeriatrics, 25(4), 517.

[29] Sheppard, J., \& Thomas, C. B. (2021). Community pharmacists and communication in the time of COVID19: applying the health belief model. Research in Social and Administrative Pharmacy, 17(1), 1984-1987.

[30] Chen, M. F., Wang, R. H., Schneider, J. K., Tsai, C. T., Jiang, D. D. S., Hung, M. N., \& Lin, L. J. (2011). Using the health belief model to understand caregiver factors influencing childhood influenza vaccinations. Journal of Community Health Nursing, 28(1), 29-40.

[31] Guvenc, G., Akyuz, A., \& Açikel, C. H. (2011). Health belief model scale for cervical cancer and Pap smear test: psychometric testing. Journal of advanced nursing, 67(2), 428-437.

[32] Meskó, B., Drobni, Z., Bényei, É., Gergely, B., \& Győrffy, Z. (2017). Digital health is a cultural transformation of traditional healthcare. Mhealth, 3.

[33] Rieke, N., Hancox, J., Li, W., Milletari, F., Roth, H. R., Albarqouni, S., ... \& Cardoso, M. J. (2020). The future of digital health with federated learning. NPJ digital medicine, 3(1), 1-7. 